\title{
Maximum number of colourings: 5-chromatic case
}

\author{
Fiachra Knox* \\ Department of Mathematics \\ Simon Fraser University \\ Burnaby, BC V5A 1S6, Canada
}

\author{
Bojan Mohar ${ }^{\dagger \ddagger}$ \\ Department of Mathematics \\ Simon Fraser University \\ Burnaby, BC V5A 1S6, Canada \\ mohar@sfu.ca
}

Submitted: May 19, 2018; Accepted: Aug 12, 2019; Published: Aug 30, 2019

(C) The authors. Released under the CC BY-ND license (International 4.0).

\begin{abstract}
In 1971, Tomescu conjectured [Le nombre des graphes connexes $k$-chromatiques minimaux aux sommets étiquetés, C. R. Acad. Sci. Paris 273 (1971), 1124-1126] that every connected graph $G$ on $n$ vertices with $\chi(G)=k \geqslant 4$ has at most $k !(k-$ $1)^{n-k} k$-colourings, where equality holds if and only if the graph is formed from $K_{k}$ by repeatedly adding leaves. In this note we prove (a strengthening of) the conjecture of Tomescu when $k=5$.
\end{abstract}

Mathematics Subject Classifications: 05C15, 05C31

\section{Introduction}

Let $x$ be a positive integer. By an $x$-colouring we mean a function $f: V(G) \rightarrow\{1, \ldots, x\}$ such that $f(u) \neq f(v)$ whenever $u v \in E(G)$. Note that permuting the colours used in a colouring gives a different colouring. The chromatic polynomial $P_{G}(x)$ is the polynomial of degree $n=|V(G)|$ whose value $P_{G}(x)$ is equal to the number of $x$-colourings of $G$ for every positive integer $x$.

Very basic questions about chromatic polynomials remain unresolved and poorly understood. We refer to part I [7] for history and motivation related to using the chromatic polynomial. In this paper we continue the work on maximizing the number of colourings among all connected graphs of given order, with the goal to prove a conjecture of Tomescu [10] dating back to 1971. The conjecture is motivated by the following easy fact. For every

\footnotetext{
* Supported by a PIMS Postdoctoral Fellowship.

†Supported in part by the NSERC Discovery Grant R611450 (Canada), by the Canada Research Chairs program, and by the Research Project J1-8130 of ARRS (Slovenia).

${ }^{\ddagger}$ On leave from IMFM, Department of Mathematics, University of Ljubljana.
} 
$k \geqslant 1$ and every integer $x \geqslant k$, every connected $n$-vertex graph containing a clique of order $k$ has at most

$$
x \cdot(x-2)(x-3) \cdots(x-k+1) \cdot(x-1)^{n-k+1}=\frac{x !}{(x-k) !}(x-1)^{n-k}
$$

$x$-colourings. This bound is attained for every $x$ if $G$ can be obtained from the $k$-clique $K_{k}$ by growing an arbitrary tree from each vertex of the clique. In 1971, Tomescu [10] conjectured that (1) is an upper bound for the number of $k$-colourings of any connected $k$-chromatic graph, whether it contains a $k$-clique or not, as long as $k \geqslant 4$ :

Conjecture 1 (Tomescu, 1971). Let $G$ be a connected $k$-chromatic graph with $k \geqslant 4$. Then $G$ has at most

$$
k !(k-1)^{|G|-k}
$$

$k$-colourings. Moreover, the extremal graphs are precisely the graphs obtained from $K_{k}$ by adding trees rooted at each vertex of the clique.

The requirement that $k \neq 3$ is necessary. Odd cycles of length at least 5 (and graphs formed from them by adding trees rooted at their vertices) have more colourings than specified by (1). See [11] for more details. In the case of bipartite graphs $(k=2)$, any connected bipartite graph attains the bound, so the bound holds but the extreme cases are plentiful.

Tomescu proved [12] that all 4-chromatic planar graphs satisfy his conjecture. For the same class of graphs, he proved a stronger conclusion for the number or $x$-colourings (for every $x \geqslant 4$ ), where the bound of the conjecture is replaced by (1). Apart from this achievement, only sporadic results are known $[2,3]$. We refer to [5, Chapter 15] for additional overview of the results in this area.

Throughout the paper we will use the related indeterminate $y=x-1$ and the shifted chromatic polynomial:

$$
Q_{G}(y)=P_{G}(y+1) .
$$

This way it is easier to handle the factor $(x-1)^{n-k+1}=y^{n-k+1}$ whose exponent depends on the number of vertices.

In [7] we proved an extended version of Tomescu conjecture for $k=4$.

Theorem $2([7])$. Let $G$ be a connected 4-chromatic graph and $y \geqslant 3$ be an integer. Then

$$
Q_{G}(y) \leqslant(y+1) y^{n-3}(y-1)(y-2) .
$$

Moreover, equality holds for some integer $y \geqslant 3$ if and only if $G$ can be obtained from $K_{4}$ by adding a tree on each vertex of $K_{4}$ (in which case equality holds for every $y \in \mathbb{R}$ ).

In this note we settle the case when $k=5$, again in its extended version for colourings with any number of colours.

Theorem 3. Let $G$ be a connected 5 -chromatic graph and $y \geqslant 4$ be an integer. Then

$$
Q_{G}(y) \leqslant(y+1) y^{n-4}(y-1)(y-2)(y-3) .
$$

Moreover, equality holds for some integer $y \geqslant 4$ if and only if $G$ can be obtained from $K_{5}$ by adding a tree on each vertex of $K_{5}$ (in which case equality holds for every $y \in \mathbb{R}$ ). 


\section{Preliminaries}

We will use standard graph theory terminology and notation as used by Diestel [4] or Bondy and Murty [1]. In particular, we use $n=|G|=|V(G)|$ to denote the order of $G$. The minimum vertex degree of $G$ is denoted by $\delta(G)$. By $N(v)$ we denote the set of neighbours of a vertex $v$, and we use $\chi(G)$ for the chromatic number. We say $G$ is $k$-chromatic if $\chi(G)=k$. The graph is vertex-critical (edge-critical) if the removal of any vertex (vertex or edge) decreases the chromatic number. If $k$ needs to be specified, we use the terms vertex/edge $k$-critical. We will frequently use the fact that identifying nonadjacent vertices of a graph $G$ results in a graph $G^{\prime}$ with $\chi\left(G^{\prime}\right) \geqslant \chi(G)$. For a vertex-set $U \subseteq V(G), G[U]$ is the subgraph of $G$ induced by $U$.

If $t$ is a positive integer, we let $[t]=\{1,2, \ldots, t\}$.

Lemma 4. Let $G$ be a graph, let $v \in V(G)$ have degree d and let $G^{\prime}=G-v$. Suppose that for every $\ell \geqslant 1$ and every graph $H$ on $\left|G^{\prime}\right|-\ell$ vertices that is formed from $G^{\prime}$ by repeatedly identifying pairs of nonadjacent vertices satisfies

$$
Q_{H}(y) \leqslant y^{-\ell} B(y)
$$

For $r \in[d-1]$, let $N_{r}=N_{r}(v)$ be the number of partitions of $N(v)$ into $r$ non-empty independent sets. Then

$$
Q_{G}(y) \leqslant(y-r) Q_{G^{\prime}}(y)+N_{r} y^{-d+r} B(y) .
$$

Proof. For each $i \in[d]$, let $Q_{i}$ be the number of $(y+1)$-colourings of $G^{\prime}$ which take exactly $i$ colours on $N(v)$. Each such colouring can be extended to a colouring of $G$ in $y-i+1$ ways. Thus,

$$
\begin{aligned}
Q_{G}(y) & =\sum_{i=1}^{d}(y-i+1) Q_{i} \\
& \leqslant \sum_{i=1}^{d}(y-r) Q_{i}+\sum_{i=1}^{d}(r-i+1) Q_{i} \\
& \leqslant(y-r) Q_{G^{\prime}}(y)+\sum_{j=0}^{r-1}(j+1) Q_{r-j}
\end{aligned}
$$

since $\sum_{i=1}^{d} Q_{i}=Q_{G^{\prime}}(y)$. (Note that if $y<r$, then $Q_{i}=0$ for every $i>r$ and so equality holds in (5).)

Let $\Omega_{r}$ be the set of partitions $\mathcal{P}$ of $N(v)$ into exactly $r$ non-empty independent sets, and let $\Omega=\bigcup_{r=1}^{d} \Omega_{r}$. For each $\mathcal{P} \in \Omega_{r}$, let $G_{\mathcal{P}}$ be the graph on $n-d-1+r$ vertices formed from $G^{\prime}$ by identifying the vertices in each part of $\mathcal{P}$ to a single vertex, and let $G_{\mathcal{P}}^{*}$ be the graph formed from $G_{\mathcal{P}}$ by adding edges between every pair of non-adjacent identified vertices. Given a partition $\mathcal{R}$ of $N(v)$ into non-empty independent sets, we 
write $\mathcal{P} \geqslant \mathcal{R}$ if $\mathcal{P}$ refines $\mathcal{R}$. For brevity we write $Q_{\mathcal{P}}(y)$ and $Q_{\mathcal{P}}^{*}(y)$ for $Q_{G_{\mathcal{P}}}(y)$ and $Q_{G_{\mathcal{P}}^{*}}(y)$, respectively. Now

$$
\sum_{j=0}^{r-1}(j+1) Q_{r-j} \leqslant \sum_{\mathcal{P} \in \Omega_{r}} \sum_{\mathcal{P} \geqslant \mathcal{R} \in \Omega} Q_{\mathcal{R}}^{*}(y)=\sum_{\mathcal{P} \in \Omega_{r}} Q_{\mathcal{P}}(y) \leqslant N_{r} y^{-d+r} B(y),
$$

where the first inequality holds since any partition $\mathcal{R} \in \Omega_{r-j}$ can be refined into a partition into $r$ parts in at least $j+1$ ways, and hence the term $Q_{\mathcal{R}}^{*}(y)$ appears at least $j+1$ times in the sum $\sum_{\mathcal{P} \in \Omega_{r}} \sum_{\mathcal{P} \geqslant \mathcal{R} \in \Omega} Q_{\mathcal{R}}^{*}(y)$. The last inequality in (6) is obtained from the assumptions of the lemma since $G_{\mathcal{P}}$ is obtained from $G^{\prime}$ by repeatedly identifying $d-r$ pairs of vertices. The desired inequality of the lemma now follows from (5) and (6).

\section{Proof of Theorem 3}

We define a partial ordering $\unlhd_{k}$ on polynomials in $y$ by $P_{2} \unlhd_{k} P_{1}$ when every coefficient of $W(z)=\left(P_{1}-P_{2}\right)(z+k)$ is non-negative. Note that this implies that $P_{1}(y) \geqslant P_{2}(y)$ for every $y \geqslant k$. We write $\unlhd$ for $\unlhd_{4}$, as in the majority of cases we will take $k=4$.

Lemma 5. Let $G$ be an edge 5-critical graph. If there exists $S \subseteq V(G)$ such that $|S| \geqslant 5$ and $\chi(G-S) \geqslant 4$, then

$$
Q_{G}(y) \leqslant(y+1) y^{n-4}(y-1)(y-2)(y-3) .
$$

Proof. We may assume that $G-S$ is connected; indeed, if $G-S$ is not connected then we can add to $S$ the vertices of each component but one (chosen to be 4 -chromatic). We may also assume that $|S|=5$, noting that we can reduce the size of $S$ (if necessary) by repeatedly removing vertices with a neighbour outside $S$.

For each vertex $v \in S$, let $d^{\prime}(v)=4-|N(v) \cap S|$ and note that $v$ has at least $d^{\prime}(v)$ neighbours in $V(G) \backslash S$. Let $S^{\prime}$ be the subset of $S$ consisting of vertices $v$ with $d^{\prime}(v) \geqslant 2$ (i.e., those vertices with $|N(v) \cap S| \leqslant 2$ ).

Let us now consider an arbitrary subset $T \subseteq S^{\prime}$ such that $N(v) \backslash S$ is an independent set in $G$ for every $v \in T$. Our goal is to provide an upper bound, $\varphi_{T}^{\prime}=\varphi_{T}^{\prime}(y)$, on the number of colourings of $G-S$ in which $N(v) \backslash S$ is monochromatic for each $v \in T$, and is not monochromatic for each $v \in S^{\prime} \backslash T$. Theorem 2 implies that the number of colourings $\varphi_{T}$ of $G-S$ in which $N(v) \backslash S$ is monochromatic for every $v \in T$ is at most $(y+1) y^{n-\Delta^{\prime}(T)-7}(y-1)(y-2)$, where $\Delta^{\prime}(T)=\max \left\{d^{\prime}(v) \mid v \in T\right\}$ (with the convention that $\Delta^{\prime}(T)=1$ if $\left.T=\emptyset\right)$, since every such colouring can be derived from a colouring of a graph formed from $G-S$ by identifying an independent set of $\Delta^{\prime}(T)$ vertices (note that identifying an independent set preserves connectivity and does not decrease the chromatic number).

Further, for any such $T \subseteq S^{\prime}$ we can compute a polynomial upper bound $\psi_{T}=\psi_{T}(y)$ on the number of extensions of a colouring of $G-S$ to $S$, given that $N(v) \backslash S$ is not monochromatic for any $v \in S^{\prime} \backslash T$. We do this by assigning to each vertex of $S$ a set of 0,1 or 2 forbidden colours (as appropriate), computing an upper bound on the number 
of extensions by deletion-contraction (where an isolated vertex with $r$ forbidden colours is given the upper bound $y-r+1$, and contracted edges produce a vertex whose set of forbidden colours is the union of the sets of forbidden colours of the endvertices), and selecting those of the resulting polynomials (in $y$ ) which are maximal under $\unlhd$. Even though $\unlhd$ is not a total ordering, in this case it turns out that each of these maximal polynomials is unique. Let us observe that there are only finitely many values for $\psi_{T}$ to be considered since the bounds depend only on $G[S]$ and the lists of forbidden colours at each vertex of $S$.

For each $T \subseteq S^{\prime}$, let $\varphi_{T}^{\prime}$ be the number of colourings of $G-S$ in which $N(v) \backslash S$ is monochromatic for every $v \in T$, but is not monochromatic for any $v \in S^{\prime} \backslash T$. We set $\varphi_{T}^{\prime}=0$ if $T$ contains a vertex for which $N(v) \backslash S$ is not independent. Then we have $Q_{G}(y) \leqslant \sum_{T \subseteq S^{\prime}} \psi_{T} \varphi_{T}^{\prime}$. Observe that $\varphi_{T}=\sum_{T \subseteq T_{1} \subseteq S^{\prime}} \varphi_{T_{1}}^{\prime}$. For each $T \subseteq S^{\prime}$, let $\psi_{T}^{\prime}=$ $\sum_{T_{0} \subseteq T}(-1)^{\left|T \backslash T_{0}\right|} \psi_{T_{0}}$. By the inclusion-exclusion formula, we have $\psi_{T}=\sum_{T_{0} \subseteq T} \psi_{T_{0}}^{\prime}$ for each $T \subseteq S^{\prime}$. Then

$$
\sum_{T \subseteq S^{\prime}} \psi_{T} \varphi_{T}^{\prime}=\sum_{T_{0} \subseteq T_{1} \subseteq S^{\prime}} \psi_{T_{0}}^{\prime} \varphi_{T_{1}}^{\prime}=\sum_{T \subseteq S^{\prime}} \psi_{T}^{\prime} \varphi_{T}
$$

In general the polynomials $\psi_{T}^{\prime}$ may be positive or negative. Fortunately, each $\psi_{T}^{\prime}$ is either positive for every integer $y \geqslant 4$, or negative for every integer $y \geqslant 4$. Our computer program has verified this using the relation $\unlhd_{k}$ (for appropriate values of $k$ ) along with individual checks for small values of $y$. Let $\Omega_{+}$(resp. $\Omega_{-}$) be the family of sets $T \subseteq S^{\prime}$ such that $\psi_{T}^{\prime}$ is positive (resp. negative) for every integer $y \geqslant 4$. Then

$$
\begin{aligned}
Q_{G}(y) & \leqslant \sum_{T \in \Omega_{+}} \psi_{T}^{\prime}(y+1) y^{n-\Delta^{\prime}(T)-7}(y-1)(y-2) \\
& =(y+1) y^{n-11}(y-1)(y-2) \sum_{T \in \Omega_{+}} \psi_{T}^{\prime} y^{4-\Delta^{\prime}(T)} .
\end{aligned}
$$

Observe that $S^{\prime}, \Omega_{+}$and $\Omega_{-}$depend only on $G[S]$. Further, $\psi_{T}^{\prime}$ and $\Delta^{\prime}(T)$ depend only on $G[S]$ and $T$ for each $T \subseteq S^{\prime}$. For each of the 34 (unlabelled) graphs on 5 vertices, we have computed the polynomial $R(y)=\sum_{T \subset S^{\prime}} \psi_{T}^{\prime} y^{4-\Delta^{\prime}(T)}$. The results are given in Table $1 .^{1}$ In each case, the resulting polynomial satisfies $R(y) \unlhd y^{7}(y-3)$ (see Table 2), and this proves the lemma.

Lemma 6. Let $G$ be an edge 5-critical graph. If $G$ has no clique or independent set of size at least 4 , then $Q_{G}(y) \unlhd(y+1) y^{n-4}(y-1)(y-2)(y-3)$.

Proof. Since $G$ is 5-critical, removing any vertex leaves a 4-chromatic graph of order at most 12, since each colour class has size at most 3. Hence $G$ has order at most 13 . The edge 5-critical graphs on at most 12 vertices have already been listed by Royle [9];

\footnotetext{
${ }^{1}$ The computer code used can be obtained from the authors.
} 
for each one, ${ }^{2}$ we have computed its chromatic polynomial $Q$ and verified that $Q \unlhd$ $(y+1) y^{n-4}(y-1)(y-2)(y-3)$.

It remains only to check graphs on exactly 13 vertices. Using Brendan McKay's program nauty_geng, one can list all of the Ramsey $(4,4)$ graphs on 13 vertices. In fact these were already listed by McKay [8]. We have tested each such graph for edge 5-criticality. Only 525 such graphs are edge 5-critical. Again, for each such graph we have computed its chromatic polynomial $Q$. As it turns out in all cases, we have $Q \unlhd$ $(y+1) y^{n-4}(y-1)(y-2)(y-3)$.

Proof of Theorem 3. It is easy to see that we may assume that $G$ is edge 5 -critical. (The reader may also check [7] for details.) The list of all 5-critical graphs with at most 8 vertices is available from [9]. Their chromatic polynomials satisfy the theorem. This was checked by computer. Hereafter we may therefore assume that $|G| \geqslant 9$. By Lemma 6 we may assume that $G$ has either a clique or an independent set of size 4 . If $G$ has a clique of size 4 then we apply Lemma 5 with $S$ being the vertices outside the clique. So we may assume that $G$ has an independent set $S$ of size 4 . If $G-S$ is not vertex 4-critical then we can add a vertex to $S$ so that $G-S$ is still 4-chromatic, and apply Lemma 5 . So we may assume that $G-S$ is vertex 4-critical.

Hence $G-S$ is connected and 4-chromatic. By Theorem 2 we have

$$
Q_{G-S}(y) \leqslant(y+1) y^{n-7}(y-1)(y-2) .
$$

Label the vertices of $S$ as $v_{1}, v_{2}, v_{3}, v_{4}$. Let $G_{i}=G-\left\{v_{i+1}, \ldots, v_{4}\right\}$ for $i=0,1,2,3,4$. For each $i \in[4]$, we select a subset $U_{i} \subseteq N\left(v_{i}\right)$ of its neighbors with $\left|U_{i}\right|=4$. Note that there are 7 partitions of $U_{i}$ into two non-empty sets.

For brevity we write $Q_{0}(y)=(y+1) y^{n-9}(y-1)(y-2)$. Now, we will repeatedly apply Lemma 4. We start with $(7)$ which gives us the bound $Q_{G_{0}}(y) \leqslant y^{2} Q_{0}(y)$. Now, consider $G_{0}=G_{1}-v_{1}$. By using Lemma 4 , we obtain an upper bound on $Q_{G_{1}}(y)$. Then we will add another vertex and by the same lemma obtain an upper bound on $Q_{G_{2}}(y)$. By two more repetitions, the resulting bound will apply to the graph $G_{4}=G$.

As outlined above, we first apply Lemma 4 with $G=G_{1}, v=v_{1}, B(y)=y^{2} Q_{0}(y)$ and $r=2$ to obtain

$$
Q_{G_{1}}(y) \leqslant(y-2) y^{2} Q_{0}(y)+7 Q_{0}(y)=\left(y^{3}-2 y^{2}+7\right) Q_{0}(y) .
$$

We again apply Lemma 4 , this time with $G=G_{2}, v=v_{2}, B(y)=y^{3} Q_{0}(y)$ and $r=2$ to obtain

$$
Q_{G_{2}}(y) \leqslant(y-2)\left(y^{3}-2 y^{2}+7\right) Q_{0}(y)+7 y Q_{0}(y)=\left(y^{4}-4 y^{3}+4 y^{2}+14 y-14\right) Q_{0}(y) .
$$

We apply Lemma 4 again, this time with $G=G_{3}, v=v_{3}, B(y)=y^{4} Q_{0}(y)$ and $r=1$ to obtain

$$
\begin{aligned}
Q_{G_{3}}(y) & \leqslant(y-1)\left(y^{4}-4 y^{3}+4 y^{2}+14 y-14\right) Q_{0}(y)+y Q_{0}(y) \\
& =\left(y^{5}-5 y^{4}+8 y^{3}+10 y^{2}-27 y+14\right) Q_{0}(y) .
\end{aligned}
$$

\footnotetext{
${ }^{2}$ All together there are 151948 such graphs.
} 
Finally, we apply Lemma 4 , this time with $G=G_{4}, v=v_{4}, B(y)=y^{5} Q_{0}(y)$ and $r=1$ to obtain

$$
\begin{aligned}
Q_{G_{4}}(y) & \leqslant(y-1)\left(y^{5}-5 y^{4}+8 y^{3}+10 y^{2}-27 y+14\right) Q_{0}(y)+y^{2} Q_{0}(y) \\
& =\left(y^{6}-6 y^{5}+13 y^{4}+2 y^{3}-36 y^{2}+41 y-14\right) Q_{0}(y) \\
& \leqslant\left(y^{6}-3 y^{5}\right) Q_{0}(y),
\end{aligned}
$$

as desired, where the last inequality holds since $0 \leqslant y^{6}-6 y^{5}+13 y^{4}+2 y^{3}-36 y^{2}+41 y-14 \leqslant$ $y^{6}-3 y^{5}$ for every $y \geqslant 4$.

\section{Conclusion}

The proof of the main theorem of this note relies on the 4-chromatic case proved in [7]. The main auxiliary Lemmas 5 and 6 rely on extensive case analysis and use of computer. The same method can be used for larger values of $k$. We see no difficulties of applying it for $k=6$ and possibly for a few additional values.

After submitting this paper, Fox, He, and Manners managed to prove the basic case of Tomescu Conjecture [6] for arbitrary $k$ when the number of colours is equal to $k$. Their proof uses some of our results as a basis.

\section{Acknowledgements}

We thank Jernej Azarija for his significant help and advice in writing the computer programs used to prove Lemmas 5 and 6.

\section{References}

[1] J. A. Bondy and U. S. R. Murty. Graph theory, volume 244 of Graduate Texts in Mathematics. Springer, New York, 2008.

[2] J. Brown and A. Erey. New bounds for chromatic polynomials and chromatic roots. Discrete Math., 338(11):1938-1946, 2015.

[3] J. I. Brown, A. Erey, and J. Li. Extremal restraints for graph colourings. J. Combin. Math. Combin. Comput., 93:297-304, 2015.

[4] R. Diestel. Graph theory, volume 173 of Graduate Texts in Mathematics. Springer, Heidelberg, Fourth edition, 2010.

[5] F. M. Dong, K. M. Koh, and K. L. Teo. Chromatic polynomials and chromaticity of graphs. World Scientific Publishing Co. Pte. Ltd., Hackensack, NJ, 2005.

[6] J. Fox, X. He, and F. Manners. A proof of Tomescu's graph coloring conjecture. J. Combin. Theory Ser. B, 136:204-221, 2019.

[7] F. Knox and B. Mohar. Maximum number of colourings: 4-chromatic graphs. arXiv: 1708.01781. 
[8] B. McKay. Combinatorial data. http://users.cecs.anu.edu.au/ bdm/data/ ramsey.html. Accessed: 2017-09-22.

[9] G. Royle. Colourings of small graphs. http://staffhome.ecm.uwa.edu.au/ 00013890/remote/graphs/\#cols. Accessed: 2017-09-22.

[10] I. Tomescu. Le nombre des graphes connexes $k$-chromatiques minimaux aux sommets étiquetés. C. R. Acad. Sci. Paris, 273:1124-1126, 1971.

[11] I. Tomescu. The maximum number of 3-colorings of a connected graph. Discrete Mathematics, 4(1):351-356, 1972.

[12] I. Tomescu. Maximal chromatic polynomials of connected planar graphs. Journal of Graph Theory, 14(1):101-110, 1990. 


\section{A Data tables}

\begin{tabular}{|c|c|}
\hline$G[S]$ & $R(y)=\sum_{T \subseteq S^{\prime}} \psi_{T}^{\prime} y^{4-\Delta^{\prime}(T)}$ \\
\hline $\begin{array}{c}5 K_{1} \\
K_{2} \cup 3 K_{1} \\
P_{3} \cup 2 K_{1} \\
K_{3} \cup 2 K_{1} \\
K_{1,3} \cup K_{1} \\
K_{1,4} \\
2 K_{2} \cup K_{1} \\
P_{4} \cup K_{1} \\
K_{3}+\text { leaf } \cup K_{1} \\
C_{4} \cup K_{1} \\
\overline{K_{1,4}+e} \\
K_{4} \cup K_{1} \\
P_{3} \cup K_{2} \\
\text { fork } \\
K_{1,4}+e \\
P_{5} \\
\text { bull } \\
C_{4}+\text { leaf } \\
K_{3}+\text { leaf } \cup K_{1} \\
\frac{K_{2,3}}{K_{3} \cup 2 K_{1}} \\
\frac{C_{5}}{P_{5}} \\
\frac{P_{4} \cup K_{1}}{P_{3} \cup K_{2}} \\
\frac{P_{3} \cup 2 K_{1}}{2 K_{2} \cup K_{1}} \\
K_{5}-e \\
K_{5} \\
\frac{K_{3} \cup K_{2}}{C_{4}+\text { leaf }} \overline{\text { fork }} \\
K_{1,3} \cup K_{1} \\
\overline{C_{4} \cup K_{1}}\end{array}$ & 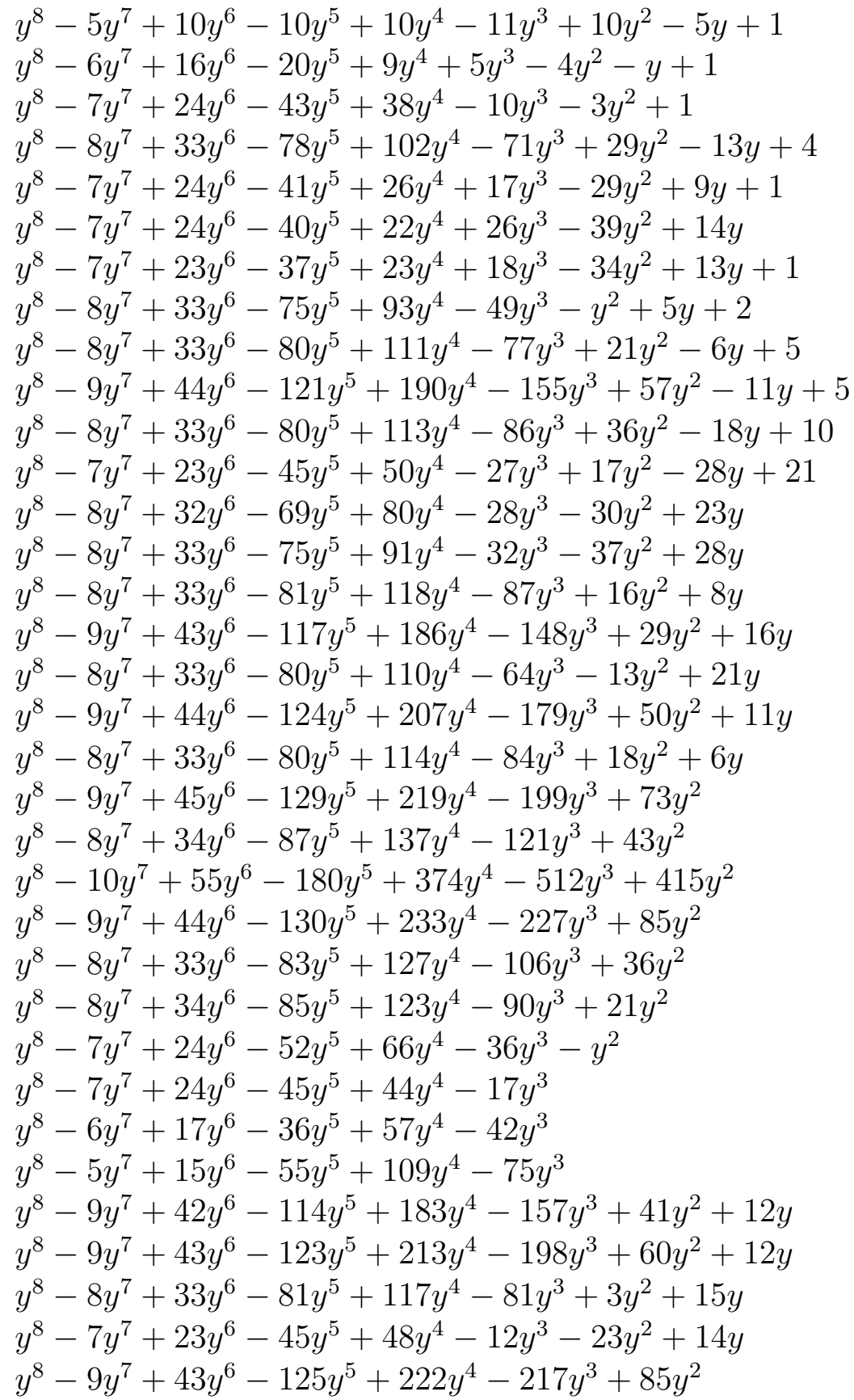 \\
\hline
\end{tabular}

Table 1: Polynomials $R(y)$ for each of the 34 graphs on 5 vertices. 


\begin{tabular}{|c|c|}
\hline$G[S]$ & $W(z)=(z+4)^{7}(z+1)-R(z+4)$ \\
\hline $5 K_{1}$ & $2 z^{7}+46 z^{6}+442 z^{5}+2270 z^{4}+6571 z^{3}+10170 z^{2}+6597 z+51$ \\
\hline$K_{2} \cup 3 K_{1}$ & $3 z^{7}+68 z^{6}+644 z^{5}+3271 z^{4}+9451 z^{3}+14952 z^{2}+10801 z+1539$ \\
\hline$P_{3} \cup 2 K_{1}$ & $4 z^{7}+88 z^{6}+811 z^{5}+4022 z^{4}+11402 z^{3}+17851 z^{2}+13048 z+2223$ \\
\hline$K_{3} \cup 2 K_{1}$ & $5 z^{7}+107 z^{6}+966 z^{5}+4738 z^{4}+13479 z^{3}+21751 z^{2}+17525 z+4640$ \\
\hline$K_{1,3} \cup K_{1}$ & $4 z^{7}+88 z^{6}+809 z^{5}+3994 z^{4}+11247 z^{3}+17425 z^{2}+12463 z+1899$ \\
\hline$K_{1,4}$ & $4 z^{7}+88 z^{6}+808 z^{5}+3978 z^{4}+11142 z^{3}+17071 z^{2}+11850 z+1464$ \\
\hline $2 K_{2} \cup K_{1}$ & $4 z^{7}+89 z^{6}+829 z^{5}+4157 z^{4}+11934 z^{3}+18986 z^{2}+14243 z+2667$ \\
\hline$P_{4} \cup K_{1}$ & $5 z^{7}+107 z^{6}+963 z^{5}+4687 z^{4}+13121 z^{3}+20461 z^{2}+15155 z+2874$ \\
\hline$K_{3}+$ leaf $\cup K_{1}$ & $5 z^{7}+107 z^{6}+968 z^{5}+4769 z^{4}+13661 z^{3}+22247 z^{2}+18126 z+4867$ \\
\hline$C_{4} \cup K_{1}$ & $6 z^{7}+124 z^{6}+1081 z^{5}+5110 z^{4}+13915 z^{3}+21067 z^{2}+14931 z+2391$ \\
\hline$\overline{K_{1,4}+e}$ & $5 z^{7}+107 z^{6}+968 z^{5}+4767 z^{4}+13638 z^{3}+22148 z^{2}+17938 z+4734$ \\
\hline$K_{4} \cup K_{1}$ & $4 z^{7}+89 z^{6}+837 z^{5}+4290 z^{4}+12827 z^{3}+22003 z^{2}+19364 z+6155$ \\
\hline$P_{3} \cup K_{2}$ & $5 z^{7}+108 z^{6}+981 z^{5}+4820 z^{4}+13628 z^{3}+21486 z^{2}+16153 z+3204$ \\
\hline fork & $5 z^{7}+107 z^{6}+963 z^{5}+4689 z^{4}+13136 z^{3}+20485 z^{2}+15116 z+2784$ \\
\hline$K_{1,4}+e$ & $5 z^{7}+107 z^{6}+969 z^{5}+4782 z^{4}+13719 z^{3}+22340 z^{2}+18120 z+4768$ \\
\hline$P_{5}$ & $6 z^{7}+125 z^{6}+1101 z^{5}+5274 z^{4}+14612 z^{3}+22675 z^{2}+16840 z+3312$ \\
\hline bull & $5 z^{7}+107 z^{6}+968 z^{5}+4770 z^{4}+13664 z^{3}+22221 z^{2}+18003 z+4732$ \\
\hline$C_{4}+$ leaf & $6 z^{7}+124 z^{6}+1084 z^{5}+5153 z^{4}+14147 z^{3}+21650 z^{2}+15605 z+2676$ \\
\hline$\overline{K_{3}+\text { leaf } \cup K_{1}}$ & $5 z^{7}+107 z^{6}+968 z^{5}+4766 z^{4}+13620 z^{3}+22046 z^{2}+17706 z+4552$ \\
\hline$K_{2,3}$ & $6 z^{7}+123 z^{6}+1065 z^{5}+5001 z^{4}+13495 z^{3}+20075 z^{2}+13576 z+1584$ \\
\hline$\overline{K_{3} \cup 2 K_{1}}$ & $5 z^{7}+106 z^{6}+951 z^{5}+4643 z^{4}+13129 z^{3}+20897 z^{2}+16216 z+3728$ \\
\hline$C_{5}$ & $7 z^{7}+141 z^{6}+1212 z^{5}+5706 z^{4}+15648 z^{3}+24353 z^{2}+18696 z+4112$ \\
\hline$\overline{P_{5}}$ & $6 z^{7}+124 z^{6}+1090 z^{5}+5247 z^{4}+14739 z^{3}+23535 z^{2}+18664 z+4720$ \\
\hline$\overline{P_{4} \cup K_{1}}$ & $5 z^{7}+107 z^{6}+971 z^{5}+4813 z^{4}+13914 z^{3}+22964 z^{2}+19136 z+5440$ \\
\hline$\overline{P_{3} \cup K_{2}}$ & $5 z^{7}+106 z^{6}+949 z^{5}+4617 z^{4}+13002 z^{3}+20611 z^{2}+15928 z+3632$ \\
\hline$\overline{P_{3} \cup 2 K_{1}}$ & $4 z^{7}+88 z^{6}+820 z^{5}+4174 z^{4}+12420 z^{3}+21233 z^{2}+18632 z+5904$ \\
\hline$\overline{2 K_{2} \cup K_{1}}$ & $4 z^{7}+88 z^{6}+813 z^{5}+4056 z^{4}+11633 z^{3}+18636 z^{2}+14384 z+3136$ \\
\hline$K_{5}-e$ & $3 z^{7}+67 z^{6}+636 z^{5}+3303 z^{4}+10010 z^{3}+17304 z^{2}+15072 z+4480$ \\
\hline$K_{5}$ & $2 z^{7}+41 z^{6}+367 z^{5}+1871 z^{4}+5851 z^{3}+11044 z^{2}+11280 z+4544$ \\
\hline$K_{3} \cup K_{2}$ & $6 z^{7}+126 z^{6}+1122 z^{5}+5457 z^{4}+15469 z^{3}+24979 z^{2}+20252 z+5504$ \\
\hline$\overline{C_{4}+\text { leaf }}$ & $6 z^{7}+125 z^{6}+1107 z^{5}+5367 z^{4}+15190 z^{3}+24492 z^{2}+19764 z+5264$ \\
\hline$\overline{\text { fork }}$ & $5 z^{7}+107 z^{6}+969 z^{5}+4783 z^{4}+13729 z^{3}+22377 z^{2}+18185 z+4820$ \\
\hline$\overline{K_{1,3} \cup K_{1}}$ & $4 z^{7}+89 z^{6}+837 z^{5}+4292 z^{4}+12844 z^{3}+22055 z^{2}+19434 z+6200$ \\
\hline$\overline{C_{4} \cup K_{1}}$ & $6 z^{7}+125 z^{6}+1109 z^{5}+5398 z^{4}+15385 z^{3}+25111 z^{2}+20744 z+5872$ \\
\hline
\end{tabular}

Table 2: All coefficients of $W(z)$ are non-negative, thus showing that $R(y) \unlhd y^{7}(y-3)$ for each graph in the table. 